\title{
Different Models and Approaches of Textual Entailment Recognition
}

\author{
Mohamed H. Haggag \\ Computer Science Department \\ Faculty of Computers and \\ Information \\ Helwan University, \\ Cairo, Egypt
}

\author{
Marwa M.A. ELFattah \\ Computer Science Department \\ Faculty of Computers and \\ Information \\ Helwan University, \\ Cairo, Egypt
}

\author{
Ahmed Mohammed \\ Ahmed \\ Ministry of Communication and \\ information Technology \\ Telecom Egypt Company, \\ Egypt \\ Giza, Egypt
}

\begin{abstract}
Variability of semantic expression is a fundamental phenomenon of a natural language where same meaning can be expressed by different texts. The process of inferring a text from another is called textual entailment. Textual Entailment is useful in a wide range of applications, including question answering, summarization, text generation, and machine translation. The recognition of textual entailment is one of the recent challenges of the Natural Language Processing (NLP) domain. This paper summarizes key ideas from the area of textual entailment recognition by considering in turn the different recognition models. The paper points to prominent testing data, training data, resources and Performance Evaluation for each model. Also this paper compares between textual entailment models according to the method which used, the result of each method and the strong and weakness of each method.
\end{abstract}

\section{Keywords}

Text entailment recognition; WordNet; Semantic analysis. Data Mining

\section{INTRODUCTION}

In natural language a single text can hold several meanings; also the same meaning can be expressed by different texts. Which means that there is a many-to-many mapping relation between language expressions and meanings. Interpreting a text correctly would, in theory, require a thorough semantic interpretation into a logic-based representation of its meanings.

Consequently, the importance of Textual Entailment Recognition have emerged since 2005 as a generic task that captures major semantic inference needs across many natural language processing applications such as text summarization (SUM), Information Retrieval (IR), Question Answering (QA), Information Extraction (IE), and Machine Translation (MT). So, recognizing textual entailment (RTE) has been a popular area of research in the last years.

Textual entailment (TE) is a directional relation between text segments. The relation holds whenever the truth of one text fragment follows from another text. The two segments are called text $(\mathrm{T})$ and hypothesis $(\mathrm{H})$. The Text $(\mathrm{T})$ is said entailed hypothesis $(\mathrm{H})$ if, typically, a human reading $t$ would infer that $\mathrm{h}$ is most likely true. This relation between $\mathrm{T}, \mathrm{H}$ is denoted as $\mathrm{T} \rightarrow \mathrm{H}$. The relation is directional because even if " $t$ entails $h "$ ", the reverse "h entails t" is much less certain.

For example, the next sentences, where the slots $\mathrm{X}$ and $\mathrm{Y}$ can be filled in with arbitrary phrases; e.g., $\mathrm{X}=$ "Jules Verne" and $\mathrm{Y}=$ "Around the World in Eighty Days".
(1) X wrote $Y$

(2) Y was written by X. (3) X is the writer of $Y$.

It is called "X wrote $\mathrm{Y} \approx \mathrm{X}$ is the author of $\mathrm{Y}$ " an inference rules -as shown in fig. 1 .

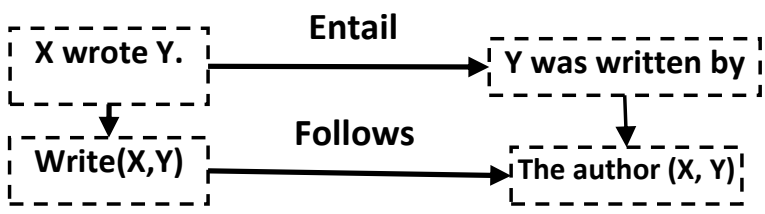

Fig 1: textual entailment Example

The formal mathematical definition for text entailment is showed in the next equation.

$$
\mathrm{p} \text { ( } \mathrm{h} \text { is TRUE } \mid \mathrm{T})>\mathrm{p} \text { ( } \mathrm{h} \text { is TRUE) }
$$

$\mathrm{P}$ ( $\mathrm{h}$ is true $\mid \mathrm{t}$ ) is the Entailment Confidence and can be considered as a measure of surety of entailment.

\section{Text entailment approaches}

A spectrum of approaches has been proposed for Recognizing Textual Entailment (RTE). Most of RTE systems are based on Machine Learning, lexical or semantic approaches [3]. However, the entailment decision problem can be considered as a classification problem. Such systems use features such as lexical, syntactic and semantic features. A common general approach for RET shown in the fig. 2, It is re-represent both the text and hypothesis and determine if the re-representation of hypothesis is subsumed by the representation of the text using comparison component the next figure shows that the text and hypothesis are represented by common way $(\Phi(\mathrm{T})$, $\Phi(\mathrm{H}))$.

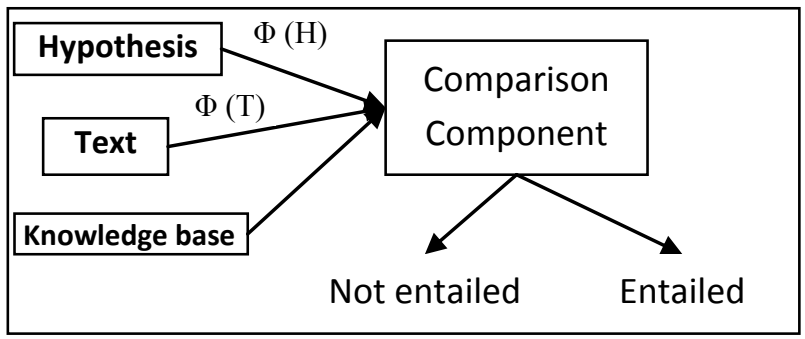

Fig 2: General Textual entailment strategy

The typical approach for recognizing textual entailment is usually made up of three main components: a representation component, a comparison component, and a decision 
component. Some models add pre-processing component before representation component.

- The representation component involves the representation of the text and the hypothesis in a way to facilitate the comparison between the two. A text can be represented as a tree, a set of predicate argument structures, a logical form, or with other representations [5].

- The comparison component compares the representation of the text and hypothesis which depends on text representation. Such as using lexical similarity for comparing between the text and hypothesis.

- The decision component is used to decide if the text is entailed the hypothesis or not depending on the comparison component output.

\subsection{Lexical Approach}

Lexical approach works directly on the input surface strings. It operates solely on a string comparison between the text and the hypothesis. The task of lexical entailment is to determine the entailment between a pair of sentences on the Basis of only lexical concepts. Common approaches include word overlap, subsequence matching, longest substring using sliding window approach. A general strategy of lexical approaches is:

1. Pre-process the given texts to separate content words and unwanted words. The pre-processing phase could involve part-of-speech (POS) tagging or named-entity recognition (NER). It does not retrieve syntactic or semantic information from the text.

2. Re-represent the texts: The text $\mathrm{T}$ and the hypothesis $\mathrm{H}$ are represented in case of lexical approaches as one of the following:

- Bag-of-words: text and hypothesis are represented as a set of words.

- N-grams: text and hypothesis are represented as Sequence of $n$ tokens. Bag of words is a special case of $n$-gram, with $n=1$, known as unigrams.

3. Compare these re-represented texts for matching: The Comparison component can be a simple counting of word overlap or comparing between lexical similarity of text and hypothesis. So the comparison component depends on the representation of text and hypothesis. Sometimes the Re-representations of text and hypothesis are compared with each other to calculate the matching score which decides the entailment. Matching is carried out on the basis of the information obtained with the help of knowledge resources.

4. Decide entailment based on the matching score: this decision is either a simple set of rules on the resulted computation, or machine learning algorithm trained on similar data. Sometimes the Decision step is done based on a certain threshold (decided experimentally) over the similarity scores generated by the algorithms. The similarity scores are calculated based on text similarity. Entailment Decisions are taken only from the lexical evidences.

In general, lexical based methods perform poorly on the task of recognizing textual entailment. The main reason of this poor performance is that textual entailment is a directional relation, where the text contains more information than the hypothesis.

Some Lexical Approaches typically uses shallow lexical resource such as WordNet. These approaches as Debarghya's model [5], his model is a simple lexical based system which detects entailment based on word overlap between the Text and Hypothesis (T-H). It starts with removing the stop words from both the T-H pair as they give a wrong impression to the matching between them.

As shown in fig. 3, Debarghya's model only performs Text Entailment at the lexical level and uses some semantic analysis for input text. It has incorporated only two type of nominal co-reference. This model starts with making some preprocessing steps such as named-entity recognizer to detect named entity in text and hypothesis then detect person names if found using person name modifier. Although the text and hypothesis is ready, various kinds of matching are preformed between text and hypothesis using different resources. The test result shows many of the matches have led to the downfall of the precision value so a separate contradiction detection module is needed to enhance the precision.

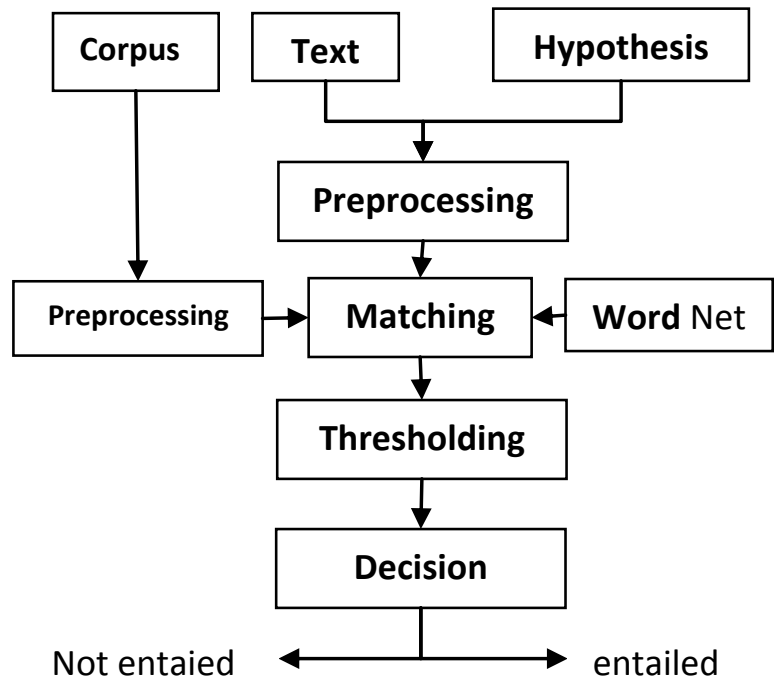

Fig 3: Debraghy's model

Adams approach [17] is another example of lexical approach. The comparison component of this method operates on a combination of word similarity measures, a web based word similarity method, and the lexical edit distance for comparing $\mathrm{T}$ and $\mathrm{a} \mathrm{H}$.

- The word similarity method used is the method that uses lexical database relations as a similarity measurement.

- The web based method is based on [18] that use web frequencies to count similarities.

- $\quad$ The lexical edit distance simply counts the number of words that were not identical from $\mathrm{H}$ to $\mathrm{T}$ relative to the length of $\mathrm{H}$, which is seen as insertion from an editing perspective.

Then the computed comparison measurements are used as an input to decision tree classifier that was trained on the development set. The classifier decides whether $\mathrm{T}$ is entailed on $\mathrm{H}$ or not. The approach achieved a relatively high accuracy of 0.63 on the RTE2 challenge. 


\subsection{Syntax Based Approach}

The most popular types of approaches for recognizing textual entailment are syntax based. Syntactic information is usually represented as directed graph. In this approach:

- The text and hypothesis are represented by a directed graph. In that graph, each word or phrase is represented by a node and the edges in the graph represent the relation between those nodes. Entailment depends upon the amount of semantic content of the given hypothesis present in the text.

- The comparison component depends on syntactic information or parse tree for the text and hypothesis. The advantage of comparing representations at a syntactic level is that it may reveal similarities that might not be evident at the linear surface level. The created trees or graphs can relate distant words, and can make the discovery of similar meaning easier even if the sentences have different word order. However, those advantages can be offset by the inaccuracies of the parsers.

- The decision component in this approach sometimes depends on similarity score between the graph of the text and hypothesis. Support vector machine or a logistic regression classifier could be used to decide if the text entails the hypothesis.

Fig. 4 shows an example for parse tree for the sentence "he watched a tiger". In this sentence, there are two nodes: "he and tiger" as noun and watched as verb. In this manner, the graph of both text and hypothesis are drawn and the similarity is calculated to reach to a final conclusion.
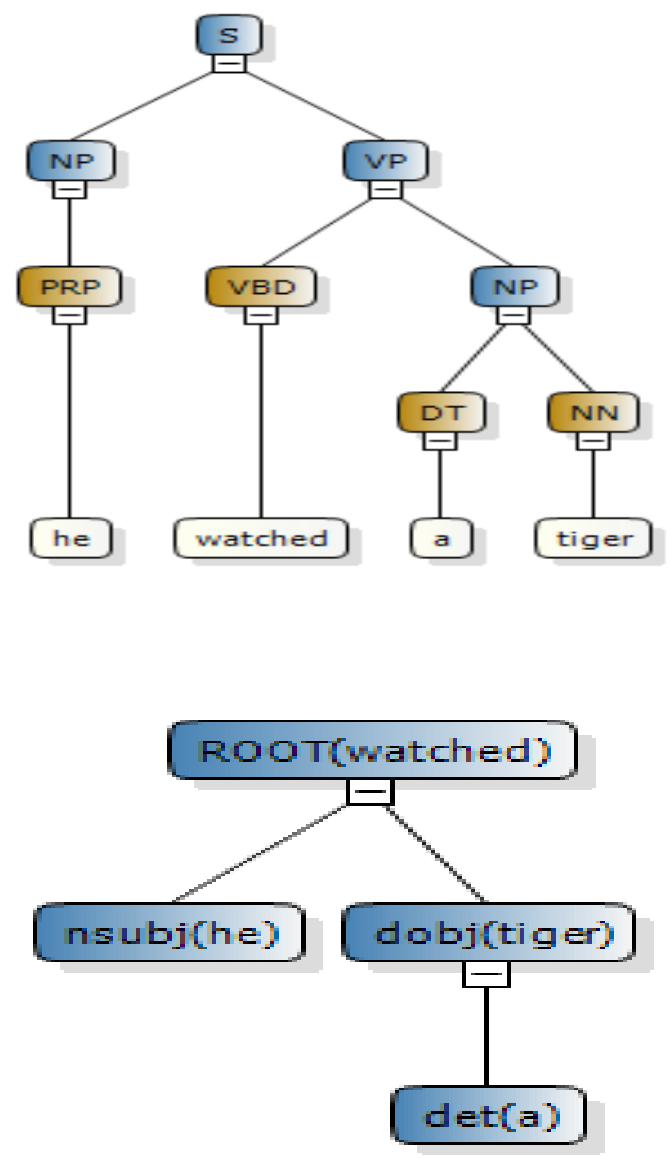

Fig 4: Parse tree for "he watched a tiger"

\subsection{Semantics-based Approach}

Semantics-based approach differs. This approach actually considers the meaning of the texts. The approach maps language expressions to semantic representations. Semantic representations are able to reveal similarities which cannot be detected by a surface or syntactic level. For example, a semantic representation can offset grammatical variability's of the same textual meaning as in the case of one semantic representation that denotes both the active and passive voice of the same text. On the other hand, the semantic analysis process might introduce inaccuracies in the representation, which might be set the advantage of revealing semantic similarities that are hard to be revealed with a syntactic based representation or a surface level one.

Semantic based methods, those that use some sort of semantic representation of the text and the hypothesis, are usually based on a predicate argument structure. In this approach:

- The text and hypothesis are represented by capturing implicit information such as information about the roles an argument plays in relation to its predicate.

- The comparison component in semantic approach compares between the representation of text and hypothesis and sometimes performs some alignment operations for the representation of text and hypothesis.

- The decision component sometimes used some rules to decide if the text entail hypothesis or not. But, the most recently approaches depend on machine learning methods.

Fig. 5 shows the William's model [18], this model extracts semantic features from the text based one new way called (UNL); it performs a lexical analysis, syntactic parsing and semantic parsing. The semantic role labeling are added using a system trained on the predicate-argument annotations. The comparison phase first performs a lexical alignment of the semantic representation, and then the generation of possible paraphrases using the web as a corpus. The decision phase is made by the extraction of dependency features, paraphrase features, and semantic features from the representation and feed it to a decision tree based machine learning classifier, which classifies the representations as either entailment or not. This approach has achieved a high accuracy of 0.75 on the RTE2 challenge.

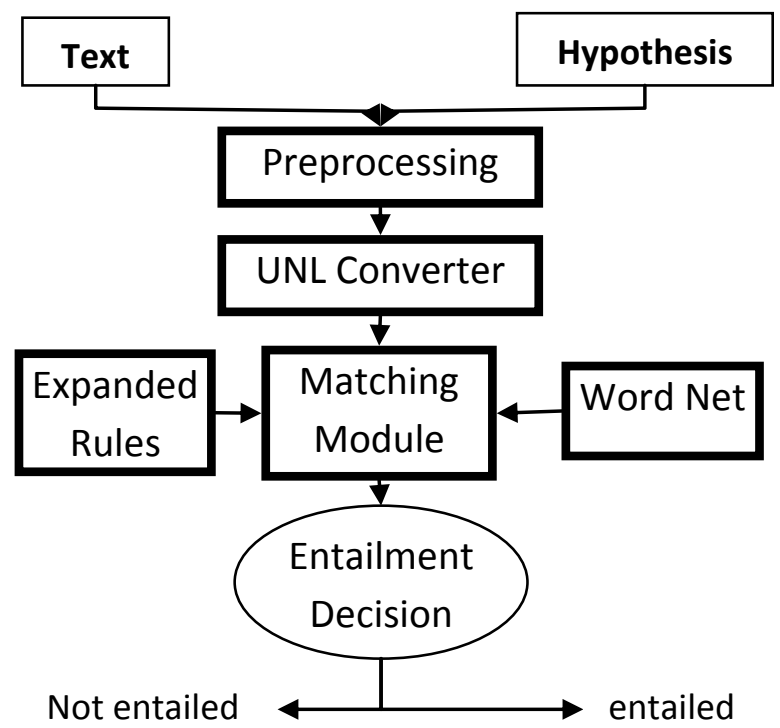

Fig 5: Wiliam's model 
On other hand, ParthaPakray model [19] introduces a two-way textual entailment (TE) recognition system that uses semantic features. It used the Universal Networking Language (UNL) to identify the semantic features. The development of a UNL based textual entailment system that compares the UNL relations in both the text and the hypothesis has been reported. The semantic TE system has been developed using the RTE-3 test annotated set as a development set (includes 800 texthypothesis pairs).The model accepts pairs of text snippets (text and hypothesis) at the input and gives a value at the output: YES if the text entails the hypothesis and NO otherwise.

Another model for semantic approach is ElisabethLien model [6] which solves the text entailment using Minimal Recursion Semantics (MRS) representations. It examines the benefits and possible disadvantages of using rich semantic representations as the basis for entailment recognition. Minimal Recursion Semantics (MRS) is a framework for computational semantics which can be used for both parsing and generation. MRS representations are expressive, have a clear interface with syntax, and are suitable for processing. An MRS representation contains a multi set of relations, called elementary predications (EPs). An EP usually corresponds to a single lexeme, but can also represent general grammatical features. An MRS has a set of handle constraints which describe how the scope arguments of the EPs can be equated with EP labels. Each EP contains a list of numbered arguments: ARG0, ARG1, etc. The value of an argument can be either a scope variable (a handle which refers to another EP's label) or a non-scope variable (events or states, or entities). The MRS for the sentence "Every tiger chases some young ox" is shown in fig. 6 .

$$
\begin{aligned}
& h 1: \operatorname{every}(x, h 3, h 4), \\
& h 3: \operatorname{tiger}(x), \\
& h 7: \text { young }(y), \\
& h 7: \text { ox }(y), \\
& h 5: \text { some }(y, h 7, h 1), \\
& h 4: \text { chase }(x, y)
\end{aligned}
$$

\section{FIG 6: MRS Example}

Sammons's model [24] is another example which creates predicate argument representations of the text and the hypothesis. They integrate multiple resources to create the representation with multiple levels of annotations. For the comparison phase, a set of entailment metrics have been devised that score the similarity of two semantic constituents. Those metrics differ from the type of annotation they are comparing. For the decision phase, the entailment metrics are used as features to a support vector machine that decides whether the text entails the hypothesis or not. This approach has achieved an accuracy of 0.64 on the RTE5 challenge.

\subsection{Logical Form Based approach}

Logical form based methods are one of the most knowledge intensive set of approaches and that relies on a logical meaning representation. A logical meaning representation is able to expose similarities that are not found in other representation levels. Such a representation is a meaning representation that is backed with a sound and understandable formal semantics and can take advantage of formal reasoning algorithms to derive information

The disadvantage of a logical based approach is the required knowledge resources in the creation of such a representation, and the needing for large number of axioms and inference rules that are not generally available. This approach relies on a manually created set of rules to do so. In addition to the resources needed, the computational processing power required is much more important than the lexical, syntactic, or semantic based approaches.

The efficiency of reasoning over a representation is directly related to the expressiveness of the representation, as such, a balance should be struck between the representation power and the reasoning capabilities. The comparison phase of such approaches relies on logical entailment usually using a theorem prover and the decision is then based on the prover's results. The main difficulty of this type of Approach is the creation of the meaning representation. Below are some of the systems that have used a logical representation in recognizing textual entailment.

Slavick's approach [11] shows a high accuracy using a logical representation and logical proving system. The logic representation is derived from a full syntactic parse, semantic parse, and a temporal representation. They then use the COGEX natural language prover originating from OTTER to prove the hypothesis from the represented text. A large number of axioms have been created from various external knowledge bases and used by the prover. The entailment decision is then based on the proof's computed score, which is a measurement of the kinds of axioms used in the proof and the significance of the dropped arguments and predicates. This approach has achieved a high accuracy of 0.73 on the RTE2 challenge.

Punyakanok's approach [12] presents an interesting approach that involves the induction of the representation of $\mathrm{T}$ and $\mathrm{H}$ into a hierarchical knowledge representation. The representation used is the Extended Feature Description Logic (EFDL) language. The representation is induced by machine learning based resources, including a tockenizer, lemmatizer, part of speech tagger, syntactic parser, semantic parser, named entity recognizer, and a name co-reference system. In additional, a set of rewrite paraphrasing rules were used to create 300 inference rules. An inference procedure is recursively applied to match the nodes in the representation. The matching information is then used to reformulate the recognizing of textual entailment problem in an equivalent Integer Linear Programming (ILP) problem. This approach has achieved an accuracy of 0.56 on the RTE4 challenge.

Clark and Harrison's BLUE (Boeing Language Understanding Engine) system [13] creates a logic based representation of the text and the hypothesis. The system uses a syntactic parser and a logical form generator to generate a semi-formal structure between a parse and full logic. The structure is a normalized tree structure with logic type elements generated by grammar rules. The semi-formal structure is then used to generate ground logical assertions by applying a set of syntactic rewrite rules recursively to the structure. The entailment task is then reduced to inferring if the hypothesis subsumes the text. The system tries to do the inference on the created representation based on inference rules that are generated from the word's logical definitions and a paraphrasing database. This approach has achieved an accuracy of 0.56 on the RTE4 challenge.

\subsection{Hybrid approach}

Hybrid methods cover approaches that use a combination of methods to recognize textual Entailment. Hybrid approaches are usually based on only two methods with one act as primary strategy and the other as a backup. However, some 
are based on multiple methods with voting mechanism to select the best result.

This approach has the capabilities of aggregating several methods and choosing the best of their results. Consequently, it should have an advantage over individual methods, yet the decision of which method result to choose from is not an easy problem to solve and require a great deal of training to be able to do so.

Wang's approach [14] creates multiple modules that each work on a specific RTE problem, and then combine the results using a voting mechanism. The modules created include:

- a time anchoring module that detects entailment relationships between temporal expressions,

- a named entity oriented module that detects entailment relationships between named entities,

- a tree skeleton module that uses a kernel based machine learning method to make the entailment prediction on dependency trees.

Different confidence values assigned to each module are used by the voting mechanism to decide on the result. If all modules fail to return a result, a backup Module that is based on a simple bag of word approach is then used. This approach achieved a high accuracy of 0.70 on the RTE4 challenge.

Bos's approach [15] combines two modules, one based on a bag of words and the other based on logical representation and inference. For the first method word overlap and word weight that are calculated as the inverse document frequency from the web are used to compute relatedness. On the other hand, the second module uses a first order fragment of the DRS language used in Discourse Representation [16] and the Vampire 7 theorem prover. A decision tree model is then created to decide which result of the two different modules to use. This approach has achieved a precision of 0.61 on the RTE2 challenge.

Fig. 7 shows the Rongyue's model [10] based on lexical similarities and an approach based on the classifier of support vector machine. The approach based on lexical similarities is to use the similarities between a set of words within a text and a set of words within a hypothesis. The approach based on the classifier means to treat this task as a classification problem. The framework of Rongyue's textual entailment recognition includes a pre-processing module in which texts and hypothesis terms are processed using Stanford CoreNLP and ClearNLP tools. Stanford CoreNLP is used for tokenization, stem, part of speech tagging, while ClearNLP is applied to identify semantic roles of sentences within texts and hypothesis.

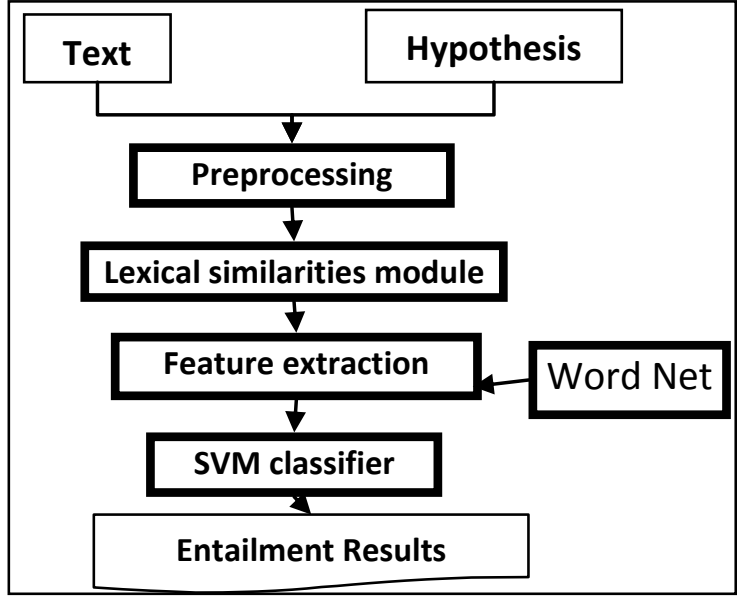

Fig 7: Rongyue's Model

Partha's model [9] is another example for a hybrid model. In which, as shown in fig. 8, a two-way Textual Entailment (TE) recognition system has been involved. One way uses lexical features and the other used syntactic features. The TE system is rule based that uses lexical and syntactic similarities. The lexical similarity features that are used in this system are: WordNet based unigram match, bi-gram match, longest common sub-sequence, skip-gram, stemming. But, in the syntactic TE system, the features used are: subject-subject comparison, subject-verb comparison, object-verb comparison and cross subject-verb comparison. The system has been separately trained on each development corpus released as part of the Recognizing Textual Entailment (RTE) competitions RTE-1, RTE-2, RTE-3 and RTE-5 and tested on the respective RTE test sets.

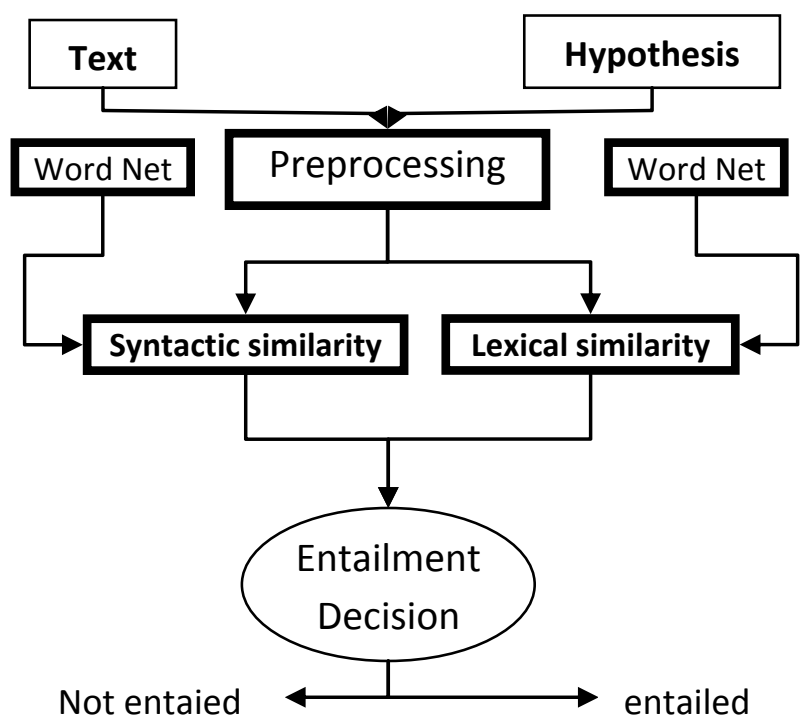

Fig 8: partha's model

There are many methods are used in decision components. However, machine learning techniques are the most commonly used in this phase. Such as Z. Zhang's model [24] which uses lexical and syntactic with semantic matching features, these features are combined together with machine learning algorithm. The method used the syntactic trees and syntactic matching similarity. All features are used in a voting style under different machine learning methods to predict whether the text sentence can entail the hypothesis sentence in a text- hypothesis pair. The experimental result shows that the 
clipping on syntactic tree structure is effective for Chinese textual entailment reorganization.

Fabio's model [23] introduces a solution to the above problem, by using a new type of feature space, which allows learning algorithms to exploit the relations between a text $(\mathrm{T})$ and a hypothesis $(\mathrm{H})$. This model proposes a classification of feature spaces in four types: the similarity, the entailment trigger, the content, and the pair content feature spaces, and uses rewrite rules to detect entailment. Many approaches to RTE rely on rewrite rules to detect entailment between text and hypothesis. Such rules are built at different linguistic levels: lexical, syntactic, and semantic. Fabio's model aims at drawing a second important distinction, between ground and first-order rewrite rules. The former are rules that do not allow the use of variables, while the latter do.

\section{TEXT ENTAILMENT MODELS ANALYSIS}

In this section, some of most important textual entailment models are analyzed according to some points of comparison. That is to build a complete view for each model, and extract their points of strength and weakness. The models which are analyzed are identified on table 1 .

While, table 2 summarize each model methodology, it describes the different models parameters, such as, using semantic analysis if this parameter is yes this mean this model uses semantic analysis if no this mean that this approach do not uses semantic analysis. Some models uses lexical and semantic analysis. Also for machine learning, three parameters are used which determine the learning behavior for the model. The first parameter decides if the model uses machine learning or not, the second decides if the machine learning algorithm can be changed without changing in the model steps, and the last one determine the machine leaning algorithm which is used.

Table 3 shows the results of each model, the parameters is divided into three fields:

- The first is experiments data which include training and testing data.

- The second field determines if the testing is executed more than one time on different data, and the methodology that implement the execution on the test data for example the first approach uses Thresholding which divided the test data into three groups and uses threshold value for each group.

- The last field determines the accuracy values for each approach, the Precision, Recall, F-Score values.

The results show that, models which use machine learning algorithms have results better than others especially if they have the ability to change their machine learning algorithms as shown in MOD 6 (A Hybrid Approach to Textual Entailment Recognition).

Table 1: the analyzed models

\begin{tabular}{|c|c|c|}
\hline Model Name & Description & ID \\
\hline $\begin{array}{l}\text { Lexical Based Text Entailment System for } \\
\text { Main Task of RTE6. }\end{array}$ & $\begin{array}{l}\text { Describes a simple lexical based system which detects entailment based on word overlap } \\
\text { between the Text and Hypothesis. }\end{array}$ & MOD 1 \\
\hline $\begin{array}{l}\text { Semantic Textual Entailment Recognition } \\
\text { uses UNL. }\end{array}$ & $\begin{array}{l}\text { A two-way textual entailment (TE) recognition system that uses semantic features has been } \\
\text { described in this paper. It used the Universal Networking Language (UNL) to identify the } \\
\text { semantic features. }\end{array}$ & MOD 2 \\
\hline $\begin{array}{l}\text { Using Minimal Recursion Semantics for } \\
\text { Entailment Recognition }\end{array}$ & $\begin{array}{l}\text { It solves the problem of textual Recognition using Minimal Recursion Semantics (MRS) } \\
\text { representations for the task of recognizing textual entailment }\end{array}$ & MOD 3 \\
\hline $\begin{array}{l}\text { Chinese Textual Entailment Recognition } \\
\text { Based on Syntactic Tree Clipping }\end{array}$ & $\begin{array}{l}\text { This model presents a novel statistical method for recognizing Chinese textual entailment in } \\
\text { which lexical, syntactic with semantic matching features are combined together. }\end{array}$ & MOD 4 \\
\hline $\begin{array}{l}\text { Statistical Relational Learning to } \\
\text { Recognize Textual Entailment. }\end{array}$ & $\begin{array}{l}\text { This model proposes a novel approach to recognize textual entailment (RTE) following two- } \\
\text { stage architecture - alignment and decision - where both stages are based on semantic } \\
\text { representations. }\end{array}$ & MOD 5 \\
\hline $\begin{array}{l}\text { A Hybrid Approach to Textual Entailment } \\
\text { Recognition }\end{array}$ & $\begin{array}{l}\text { This technique includes an approach based on lexical similarities and semantic similarities } \\
\text { and an approach based on the classifier of support vector machine. }\end{array}$ & MOD 6 \\
\hline $\begin{array}{l}\text { Textual Entailment Using Lexical and } \\
\text { Syntactic Similarity }\end{array}$ & $\begin{array}{l}\text { It uses lexical and syntactic features has been described in this paper. The TE system is rule } \\
\text { based that uses lexical and syntactic similarities. }\end{array}$ & MOD 7 \\
\hline
\end{tabular}

Table 2: the model's methodology

\begin{tabular}{|c|c|c|c|c|c|c|c|c|}
\hline \multirow{2}{*}{ ID } & \multicolumn{3}{|c|}{ Machine Learning } & \multirow{2}{*}{ Corpus } & \multirow{2}{*}{$\begin{array}{l}\text { lexical } \\
\text { analysis }\end{array}$} & \multirow{2}{*}{$\begin{array}{l}\text { Semantic } \\
\text { analysis }\end{array}$} & \multirow{2}{*}{$\begin{array}{l}\text { Syntactic } \\
\text { analysis }\end{array}$} & \multirow{2}{*}{ WORD NET } \\
\hline & $\begin{array}{l}\text { Using Machine } \\
\text { Learning }\end{array}$ & $\begin{array}{l}\text { Can change Learning } \\
\text { algorithm }\end{array}$ & $\begin{array}{l}\text { Machine Learning } \\
\text { Algorithm }\end{array}$ & & & & & \\
\hline MOD 1 & NO & NO & & YES & YES & YES & NO & YES \\
\hline MOD 2 & NO & NO & & NO & NO & YES & NO & YES \\
\hline MOD 3 & NO & NO & & NO & NO & YES & NO & NO \\
\hline MOD 4 & YES & YES & naïve Bayes & NO & NO & NO & YES & NO \\
\hline
\end{tabular}




\begin{tabular}{|l|l|l|l|l|l|l|l|l|}
\hline MOD 5 & YES & NO & & NO & YES & YES & NO & NO \\
\hline MOD 6 & YES & YES & SVM & NO & YES & YES & YES & YES \\
\hline MOD 7 & NO & NO & & NO & YES & NO & YES & YES \\
\hline
\end{tabular}

Table 3: the results of models

\begin{tabular}{|c|c|c|c|c|c|c|c|}
\hline \multirow{3}{*}{ MOD ID } & \multicolumn{7}{|c|}{ Experiments and Testing } \\
\hline & \multicolumn{2}{|c|}{ experiments Data (Pairs) } & \multicolumn{2}{|c|}{ Multiple Running } & \multicolumn{3}{|c|}{ Performance Evaluation } \\
\hline & Training Data & Testing Data & Used & Methodology & Precision & Recall & F-Score \\
\hline 1 & Not determined & Not determined & YES & Thresholding & $53.43 \%$ & $42.86 \%$ & $47.56 \%$ \\
\hline 2 & 800 & 1000 & YES & Two baseline (Yes-NO) & $60.30 \%$ & $60.30 \%$ & $63 \%$ \\
\hline 3 & 66 & 301 & NO & NO & $71 \%$ & $50 \%$ & $58.3 \%$ \\
\hline 4 & Not determined & 781 & YES & Two systems NLPWM-01 and NLPWM-02 & $71.45 \%$ & $39.12 \%$ & $74.67 \%$ \\
\hline 5 & Not determined & Not determined & YES & RT1,RT2,RT3 & $54 \%$ & $70 \%$ & $61 \%$ \\
\hline 6 & Not determined & Not determined & YES & RTE1_DEV, RTE2_DEV and RTE3_DEV & $72 \%$ & $72 \%$ & $72 \%$ \\
\hline 7 & 1087 & 1600 & YES & $\mathrm{RT} 1, \mathrm{RT} 2$ & $57 \%$ & $57 \%$ & $57 \%$ \\
\hline
\end{tabular}

\section{REFERENCES}

[1] Russell, Stuart and P.Norvig. "Artificial Intelligence: A Modern Approach". Prentice-Hall, Englewood Cliffs, NJ.1995

[2] S.Gautam , "Text Entailment and Machine Translation Evaluation", Center for Indian Language Technology (CFILT) ,2014

[3] I.Androutsopoulos , P. Malakasiotis , "A Survey of Paraphrasing and Textual Entailment Methods", Journal of Artificial Intelligence Research 38 (2010) 135-187, Submitted 12/09; published 05/10.

[4] I.DAGAN, B.DOLAN, B.MAGNINI and D.ROTH , "Recognizing textual entailment: Rational, evaluation and approaches" - Erratum , Natural Language Engineering / Volume 16 / Issue 01 / January 2010, pp 105 - 105 DOI: 10.1017/S1351324909990234, Published online: 28 January 2010 .

[5] D. Majumdar , P.Bhattacharyya ,"Lexical Based Text Entailment System for Main Task of RTE6", Text Analysis Conference (TAC) Proceedings ,2010

[6] E.Lien , "Using Minimal Recursion Semantics for Entailment Recognition ", Proceedings of the Student Research Workshop at the 14th Conference of the European Chapter of the Association for Computational Linguistics, pages 76-84, Gothenburg, Sweden, April 26-30 2014. c 2014 Association for Computational Linguistics.
[7] Z.Zhang, D.Yao, S.Chen, and H.Ma , "Chinese Textual Entailment Recognition Based on Syntactic Tree Clipping", M. Sun et al. (Eds.): CCL and NLP-NABD 2014, LNAI 8801, pp. 83-94, 2014.@ Springer International Publishing Switzerland 2014.

[8] M.Rios, L.Specia, A.Gelbukh, and R.Mitkov, "Statistical Relational Learning to Recognise Textual Entailment" , A. Gelbukh (Ed.): CICLing 2014, Part I, LNCS 8403, pp. 330-339, 2014,_c Springer-Verlag Berlin Heidelberg 2014.

[9] P.Pakray, S.Bandyopadhyay and A.Gelbukh, "TEXTUAL ENTAILMENT USING LEXICAL AND SYNTACTIC SIMILARITY", International Journal of Artificial Intelligence \& Applications (IJAIA), Vol.2, No.1, January 2011 .

[10] R.Mei ，X. Li , "A Hybrid Approach to Textual Entailment Recognition", International Conference on Mechatronics, Electronic, Industrial and Control Engineering (MEIC 2014).

[11] M. Tatu, B. Iles, J. Slavick, A. Novischi, and D. Moldovan." Cogex at the second recognizing textual entailment challenge". In Proceedings of the Second PASCAL Challenges Workshop on Recognising Textual Entailment, pages 104\{109, Venice, Italy, April 2006.

[12] R.de Salvo Braz, R. Girju, V. Punyakanok, D. Roth, and M. Sammons. "An inference model for semantic entailment in natural language". Machine Learning Challenges: Evaluating Predictive Uncertainty, Visual 
Object Classification, and Recognising Textual Entailment, pages 261\{286, 2006.

[13] P. Clark and P. Harrison. "Recognizing textual entailment with logical inference". In Proceedings of the 2008 Text Analysis Conference (TAC'08), Gaithersburg, Maryland, USA, November 2008.

[14] R.Wang and G.Neumann." A divide-and-conquer strategy for recognizing textual entailment". Text Analysis Conference (TAC'08), Gaithersburg, Maryland, USA, November 2008.

[15] J.Bos and K.Markert. "When logical inference helps determining textual entailment" (and when it doesn't). In Proceedings of the Second PASCAL RTE Challenge, Venice, Italy, April 2006.

[16] A.Riazanov and A.Voronkov. "The design and implementation of vampire". AI communications, 15(2):91\{110, 2002.

[17] R. Adams. "Textual entailment through extended lexical overlap". In Proceedings of the Second PASCAL Challenges Workshop on Recognising Textual Entailment, pages 128\{133, Venice, Italy, April 2006.

[18] G. Hirst and D. St-Onge. "Lexical chains as representations of context for the detection and correction of malapropisms". WordNet An electronic lexical database, pages 305\{332, April 1998.
[19] A. Iftene. "UAIC Participation at RTE4". Text Analysis Conference (TAC'08), Gaithersburg, Maryland, USA, November 2008.

[20] D.Lin. "Dependency-based evaluation of minipar". Treebanks, pages 317\{329, 2003.

[21] F.M. Zanzotto, A. Moschitti, M. Pennacchiotti, and M.T. Pazienza. "Learning textual entailment from examples". In Proceedings of the Second PASCAL Challenges Workshop on Recognising Textual Entailment, Venice, Italy, April 2006.

[22] M.C. de Marne e, B. MacCartney, T. Grenager, D. Cer, A. Ra_erty, and C.D. "Manning. Learning to distinguish valid textual entailments". In Proceedings of the Second PASCAL RTE Challenge Workshop, Venice, Italy, April 2006.

[23] R. Bar-Haim, J. Berant, and I. Dagan. "A compact forest for scalable inference over entailment and paraphrase rules". In Proceedings of the 2009 Conference on Empirical Methods in Natural Language Processing: Volume 3, pages 1056\{1065, Singapore, August 2009. Association for Computational Linguistics.

[24] M. Sammons, V.G.V. Vydiswaran, T. Vieira, N. Johri, M.W. Chang, D. Goldwasser, V. Srikumar, G. Kundu, Y. $\mathrm{Tu}, \mathrm{K}$. Small, et al. Relation "alignment for textual entailment recognition". In Proceedings of the 2009 Text Analysis Conference (TAC'09), Gaithersburg, Maryland, USA, November 2009. 